\title{
Notes on certain analytic functions concerning some subordinations
}

\author{
Şahsene Altinkaya, Shigeyoshi Owa, and Sibel Yalçin
}

ABSTRACT. By making use of the principle of subordination, we investigate a certain subclass of analytic functions. Such results as subordination and superordination are given. The related sandwich-type results are also presented.

\section{Introduction}

Let $\mathcal{A}$ denote the class of functions $f$ defined by

$$
f(z)=z+\sum_{n=2}^{\infty} a_{n} z^{n}
$$

which are analytic in the open unit disk $\mathbb{U}=\{z: z \in \mathbb{C},|z|<1\}$, with $f(0)=0$ and $f^{\prime}(0)=1$. Let us consider a function $g$ given by

$$
g(z)=\frac{\alpha(1-z)}{\alpha-z} \quad(z \in \mathbb{U})
$$

for some real $\alpha>1$. If we write $z=r e^{i \theta}(0 \leqq r<1,0 \leqq \theta<2 \pi)$, then $g(0)=1$ and

$$
\Re(g(z))=\frac{\alpha\left(\alpha+r^{2}-r(1+\alpha) \cos \theta\right)}{\alpha^{2}+r^{2}-2 \alpha r \cos \theta} .
$$

This gives us inequalities

$$
\frac{\alpha(1-r)}{\alpha-r} \leqq \Re(g(z)) \leqq \frac{\alpha(1+r)}{\alpha+r}<\frac{2 \alpha}{\alpha+1} .
$$

Received May 30, 2018.

2010 Mathematics Subject Classification. 30C45.

Key words and phrases. Analytic function; starlike function; convex function; subordination; superordination.

http://dx.doi.org/10.12697/ACUTM.2019.23.08 
Definition 1. Let the functions $f, g \in \mathcal{A}$ be analytic in $\mathbb{U}$. Then $f$ is said to be subordinate to $g$ if there exits a Schwarz function $w \in \Lambda$, where

$$
\Lambda=\{w: w(0)=0,|w(z)|<1\} \quad(z \in \mathbb{U})
$$

such that

$$
f(z)=g(w(z)) \quad(z \in \mathbb{U}) .
$$

We denote this subordination by

$$
f(z) \prec g(z) \quad(z \in \mathbb{U}) .
$$

In particular, if the function $g$ is univalent in $\mathbb{U}$, then the subordination (1) is equivalent to

$$
f(0)=g(0) \quad \text { and } \quad f(\mathbb{U}) \subset g(\mathbb{U}) .
$$

By using the subordination, we say that $f \in P(\alpha)(\alpha>1)$ if $f \in \mathcal{A}$ satisfies

Then, since

$$
f^{\prime}(z) \prec \frac{\alpha(1-z)}{\alpha-z} .
$$

$$
0<\Re\left(f^{\prime}(z)\right)<\frac{2 \alpha}{\alpha+1} \quad(z \in \mathbb{U})
$$

we obtain that $f \in P(\alpha)$ is close-to-convex function in $\mathbb{U}$ (see [4] or [13]).

The following results will be required in our analysis of the class $P(\alpha)$.

Lemma 1 (see [3], [7], [8]). Let $w$ be analytic in $\mathbb{U}$ with $w(0)=0$. Then, if $|w(z)|$ attains its maximum value on the circile $|z|=r<1$ at a point $z_{0} \in \mathbb{U}$, then we have that

$$
z_{0} w^{\prime}\left(z_{0}\right)=k w\left(z_{0}\right)
$$

and

$$
\Re\left(1+\frac{z_{0} w^{\prime \prime}\left(z_{0}\right)}{w^{\prime}\left(z_{0}\right)}\right) \geqq k
$$

where $k \geqq 1$.

Miller and Mocanu [9] have given the following lemma for superordinations.

Lemma 2. Let $h_{1}$ and $h_{2}$ be convex in $\mathbb{U}$ and let $f$ be univalent in $\mathbb{U}$ with $h_{1}(0)=h_{2}(0)=f(0)$. Let $\gamma \neq 0$ with $\Re(\gamma) \geqq 0$. If

$$
h_{1}(z) \prec f(z) \prec h_{2}(z),
$$

then

$$
\frac{\gamma}{z^{\gamma}} \int_{0}^{z} h_{1}(t) t^{\gamma-1} d t \prec \frac{\gamma}{z^{\gamma}} \int_{0}^{z} f(t) t^{\gamma-1} d t \prec \frac{\gamma}{z^{\gamma}} \int_{0}^{z} h_{2}(t) t^{\gamma-1} d t,
$$

when the middle integral is univalent. 
It should be remarked in passing that, in recent years, several authors obtained many interesting results associated with subordination and superordination in the unit disk. The interested reader may refer to several earlier works including, for example, [1], [2], [5], [6], [10], [11], and [12].

\section{Some properties}

We first derive the following theorem.

Theorem 1. If $f \in \mathcal{A}$ satisfies

$$
\sum_{n=2}^{\infty} n\left|a_{n}\right| \leqq \frac{\alpha-1}{\alpha+1} \quad(z \in \mathbb{U})
$$

for $\alpha>1$, then $f \in P(\alpha)$. The equality is attained for

$$
f(z)=z+\sum_{n=2}^{\infty} \frac{(\alpha-1) \varepsilon}{n^{2}(n-1)(\alpha+1)} z^{n} \quad(|\varepsilon|=1) .
$$

Proof. Let us consider a function $f \in \mathcal{A}$ which satisfies

$$
\left|\left(\alpha^{2}-6 \alpha+1\right)+4(\alpha+1) f^{\prime}(z)\right|<\left|\left(\alpha^{2}+10 \alpha+1\right)-4(\alpha+1) f^{\prime}(z)\right|
$$

for $\alpha>1$ and $f \in \mathcal{A}$. From the inequality (4), we get

$$
f^{\prime}(z)+\overline{f^{\prime}(z)}<\frac{4 \alpha}{\alpha+1}
$$

which yields

$$
0<\Re\left(f^{\prime}(z)\right)<\frac{2 \alpha}{\alpha+1} \quad(z \in \mathbb{U}) .
$$

Hence, we get $f \in P(\alpha)$.

If $f$ satisfies (4), then we have that

To satisfy (4), $f$ has to satisfy

$$
\begin{aligned}
& \left|\left(\alpha^{2}-2 \alpha+5\right)+4(\alpha+1) \sum_{n=2}^{\infty} n a_{n} z^{n-1}\right| \\
& \quad<\left|\alpha^{2}+6 \alpha-3-4(\alpha+1) \sum_{n=2}^{\infty} n a_{n} z^{n-1}\right| .
\end{aligned}
$$

$$
(\alpha+1) \sum_{n=2}^{\infty} n\left|a_{n}\right| \leqq \alpha-1 .
$$

Thus, if $f \in \mathcal{A}$ satisfies (2), then the inequality (4) is satisfied.

Further, if we consider $f$ given by (3), then we deduce that

$$
\sum_{n=2}^{\infty} n\left|a_{n}\right|=\sum_{n=2}^{\infty} \frac{(\alpha-1)}{n(n-1)(\alpha+1)}=\frac{\alpha-1}{\alpha+1} \sum_{n=2}^{\infty}\left(\frac{1}{n-1}-\frac{1}{n}\right)=\frac{\alpha-1}{\alpha+1} .
$$


The proof of theorem is thus completed.

The following theorem is on the extremal function for the class $P(\alpha)$.

Theorem 2. The extremal function $f$ for the class $P(\alpha)$ is

$$
f(z)=\alpha z+\alpha(\alpha-1) \log (\alpha-z) .
$$

Proof. It is easy to see that $f$, given by (5), satisfies

$$
f^{\prime}(z)=\alpha-\frac{\alpha(\alpha-1)}{\alpha-z}=\frac{\alpha(1-z)}{\alpha-z} .
$$

This means that $f$ given by (5) is the extremal function for the class $P(\alpha)$.

Now, we are ready to state and prove our next theorem.

Theorem 3. If $f \in P(\alpha)$ with $a_{n} \geqq 0$, then

$$
\sum_{n=2}^{\infty} n a_{n} \leqq \alpha-1 \text {. }
$$

The equality in (6) is attained for

$$
f(z)=z+\sum_{n=2}^{\infty} \frac{\alpha-1}{n^{2}(n-1)} z^{n} .
$$

Proof. If $f \in P(\alpha)$, then there exists a function $w$ which is analytic in $\mathbb{U}$, $w(0)=0,|w(z)|<1 \quad(z \in \mathbb{U})$, and

$$
f^{\prime}(z)=\frac{\alpha(1-w(z))}{\alpha-w(z)} .
$$

This shows us that

Therefore, we have

$$
|w(z)|=\left|\frac{\alpha\left(f^{\prime}(z)-1\right)}{f^{\prime}(z)-\alpha}\right|<1 .
$$

$$
\left|(\alpha+1) f^{\prime}(z)-\alpha\right|<\alpha
$$

Letting $z \rightarrow 1^{-}$in (8), we obtain that

$$
1+\sum_{n=2}^{\infty} n a_{n} \leqq \alpha
$$

which is equivalent to (6). Also, considering $f$ given by (7), we see that

$$
\sum_{n=2}^{\infty} n a_{n}=\sum_{n=2}^{\infty} \frac{\alpha-1}{n(n-1)}=(\alpha-1) \sum_{n=2}^{\infty}\left(\frac{1}{n-1}-\frac{1}{n}\right)=\alpha-1
$$


In the light of Lemma 1, we obtain the next result.

Theorem 4. If $f \in \mathcal{A}$ satisfies

$$
\Re\left(\frac{z f^{\prime \prime}(z)}{f^{\prime}(z)}\right)<\frac{\alpha-1}{2(\alpha+1)} \quad(z \in \mathbb{U})
$$

for some real $\alpha>1$, then $f \in P(\alpha)$.

Proof. Let us consider an analytic function $w$ in $\mathbb{U}$ given by

$$
f^{\prime}(z)=\frac{\alpha(1-w(z))}{\alpha-w(z)},
$$

with $w(0)=0$, and such that $f$ satisfies (9). It is easy to see that the equation (10) gives

$$
\frac{z f^{\prime \prime}(z)}{f^{\prime}(z)}=\frac{z w^{\prime}(z)}{w(z)}\left(\frac{w(z)}{\alpha-w(z)}-\frac{w(z)}{1-w(z)}\right) .
$$

Now, we suppose that there exists a point $z_{0} \in \mathbb{U}$ such that

$$
\max _{|z| \leqq\left|z_{0}\right|}|w(z)|=\left|w\left(z_{0}\right)\right|=1
$$

Then, in view of Lemma 1 , we get

$$
z_{0} w^{\prime}\left(z_{0}\right)=k w\left(z_{0}\right) \quad(k \geqq 1) .
$$

Letting $w\left(z_{0}\right)=e^{i \theta}$, we obtain that

$$
\begin{aligned}
\Re\left(\frac{z_{0} f^{\prime \prime}\left(z_{0}\right)}{f^{\prime}\left(z_{0}\right)}\right) & =k \Re\left(\frac{e^{i \theta}}{\alpha-e^{i \theta}}-\frac{e^{i \theta}}{1-e^{i \theta}}\right)=k\left(\frac{\alpha \cos \theta-1}{\alpha^{2}+1-2 \alpha \cos \theta}+\frac{1}{2}\right) \\
& \geqq \frac{\alpha-1}{2(\alpha+1)} .
\end{aligned}
$$

This contradicts the condition (9) of the theorem. Therefore, we say that there is no $z_{0} \in \mathbb{U}$ such that $\left|w\left(z_{0}\right)\right|=1$. This shows us that $|w(z)|<1$ for all $z \in \mathbb{U}$. It follows from the equation (10) that

$$
f^{\prime}(z) \prec \frac{\alpha(1-z)}{\alpha-z},
$$

which yields

$$
f \in P(\alpha)
$$

Finally, we consider a sandwich-type result for $f \in P(\alpha)$.

Theorem 5. Let $f \in \mathcal{A}$ satisfy

$$
\frac{\beta(1-z)}{\beta-z} \prec f^{\prime}(z) \prec \frac{\alpha(1-z)}{\alpha-z}
$$


for $1<\beta<\alpha$. Then we have

$$
\frac{\beta}{z}\left(z+\log \left(\frac{\beta-z}{\beta}\right)^{\beta-1}\right) \prec \frac{f(z)}{z} \prec \frac{\alpha}{z}\left(z+\log \left(\frac{\alpha-z}{\alpha}\right)^{\alpha-1}\right) .
$$

Proof. The function

$$
\log \left(\frac{\beta-z}{\beta}\right)^{\beta-1}
$$

is well-defined and analytic for $|z|<1$ by the requirement $\log 1=0$.

Now, let us define a function $g$ by

$$
g(z)=\frac{\alpha(1-z)}{\alpha-z} \quad(z \in \mathbb{U}) .
$$

Then $g$ satisfies $g(0)=1$ and

$$
1+\frac{z g^{\prime \prime}(z)}{g^{\prime}(z)}=1+\frac{2 z}{\alpha-z} .
$$

If we take $z=e^{i \theta}$, then we get

$$
\Re\left(1+\frac{z g^{\prime \prime}(z)}{g^{\prime}(z)}\right)=\Re\left(1+\frac{2 e^{i \theta}}{\alpha-e^{i \theta}}\right)=1+\frac{2(\alpha \cos \theta-1)}{\alpha^{2}+1-2 \alpha \cos \theta}>\frac{\alpha-1}{\alpha+1} .
$$

Therefore, $g$ is convex in $\mathbb{U}$. This shows us that $\frac{\beta(1-z)}{\beta-z}$ and $\frac{\alpha(1-z)}{\alpha-z}$ are convex in $\mathbb{U}$ with $1<\beta<\alpha$.

Further, we note that $f$ is close-to-convex in $\mathbb{U}$. This means that $f$ is univalent in $\mathbb{U}$. Thus, applying Lemma 2 , if $f$ satisfies the superordination (11), then, taking $\gamma=1$, we have that

$$
\frac{1}{z} \int_{0}^{z} \frac{\beta(1-t)}{\beta-t} d t \prec \frac{1}{z} \int_{0}^{z} f^{\prime}(t) d t \prec \frac{1}{z} \int_{0}^{z} \frac{\alpha(1-t)}{\alpha-t} d t
$$

It is easy to show that $f$ satisfies the superordination (12).

\section{References}

[1] R. M. Ali, V. Ravichandran, and N. Seenivasagan, On subordination and superordination of the multiplier transformation for meromorphic functions, Bull. Malays. Math. Sci. Soc. 33 (2010), 311-324.

[2] N. E. Cho and H. M. Srivastava, A class of nonlinear integral operators preserving subordination and superordination, Integral Transforms Spec. Funct. 18 (2007), 95107.

[3] I. S. Jack, Functions starlike and convex of order alpha, J. London Math. Soc. 3 (1971), 469-474.

[4] W. Kaplan, Close-to-convex schlicht functions, Michigan Math. J. 1 (1952), 169-185.

[5] S. S. Miller and P. T. Mocanu, On some classes of first order differential subordinations, Michigan Math. J. 32 (1985), 185-195.

[6] S. S. Miller and P. T. Mocanu, Differential subordinations and inequalities in the complex plane, J. Diff. Egns. 67 (1987), 199-211. 
[7] S. S. Miller and P. T. Mocanu, Briot-Bouquet differential subordinations, Complex Variables Theory Appl. 33 (1997), 217-237.

[8] S. S. Miller and P. T. Mocanu, Differential Subordinations, Theory and Applications, Mercel Dekker, Inc., New York, 2000.

[9] S. S. Miller and P. T. Mocanu, Subordinations of differential superordinations, Complex Var. Theory Appl. 48 (2003), 815-826.

[10] M. Obradović and S. Owa, On certain properties for some classes of starlike functions, J. Math. Anal. Appl. 145 (1987), 357-364.

[11] S. Owa and M. Obradović, An application of differential subordinations and some criteria for univalency, Bull. Austral. Math. Soc. 41 (1990), 487-494.

[12] S. Owa and H. M. Srivastava, Univalent and starlike generalized hypergeometric functions, Canad. J. Math. 39 (1987), 1057-1077.

[13] Ch. Pommerenke, On close-to-convex analytic functions, Trans. Amer. Math. Soc. 114 (1965), 176-186.

Department of Mathematics, Faculty of Arts and Sciences, Bursa Uludag University, 16059 Bursa, Turkey

E-mail address: sahsenealtinkaya@gmail.com

University of Alba Iulia, 510009 Alba Iulia, Romania

E-mail address: shige21@ican.zaq.ne.jp

Department of Mathematics, Faculty of Arts and Sciences, Bursa Uludag University, 16059 Bursa, Turkey

E-mail address: syalcin@uludag.edu.tr 